\title{
Modeling Nonlinearity in Coherent Transmissions with Dominant Interpulse-Four-Wave-Mixing
}

\author{
A. Bononi ${ }^{1}$, E. Grellier ${ }^{2}$, P. Serena ${ }^{1}$, N. Rossi ${ }^{1}$, and F. Vacondio ${ }^{2}$ \\ ${ }^{1}$ Dip. Ing. informazione, Università di Parma, Italy; ${ }^{2}$ Alcatel-Lucent Bell Labs, Villarceaux, France
}

\begin{abstract}
We provide a new analytical model to predict the nonlinear interference coefficient and the nonlinear threshold in coherent transmissions with dominant single-channel IFWM.
\end{abstract}

OCIS codes: (060.1660) Coherent communications, (060.4370) Nonlinear optics, fibers

\section{Introduction}

It has recently been shown that, in high bit-rate coherent optical links with no dispersion management (NDM), the nonlinear interference (NLI) is a zero-mean additive circular complex-Gaussian noise, independent of the symbol of interest, already after a few spans [1]. Based on such a powerful observation, a nonlinear Gaussian model for NDM coherent communications was proposed [2-4]. In this paper, we wish to extend those studies to the regime in which single-channel inter-pulse four wave mixing (IFWM) is the dominant nonlinearity. This regime includes both dispersion-managed (DM) and NDM links at sufficiently large baud-rates.

\section{Nonlinear Gaussian Model}

Consider a single-channel long-haul optical link with dual polarization coherent reception. Assume that both the amplified spontaneous emission (ASE) and the NLI are independent additive complex-Gaussian noises. After coherent reception with polarization demultiplexing and ideal linear electrical equalization, followed by matched filtering with ideal carrier estimation, the 2-dimensional (2D) sampled received complex field vector is: $\underline{r}(t)=\sqrt{P} \underline{U}(t)+\underline{n}_{L}(t)+$ $\underline{n}_{N L}(t)$, where $P[\mathrm{~W}]$ is the signal average power, $\underline{U}$ the normalized signal vector, $\underline{n}_{L}$ the ASE, and $\underline{n}_{N L}$ the NLI. The electrical signal-noise ratio (SNR) at the decision gate is

$$
S=\frac{P}{N_{A}+N_{N L}}
$$

where $N_{A}=\operatorname{Var}\left[\underline{n}_{L}\right]=\beta N$ is the ASE power, which linearly increases with the number of spans $N$, and $N_{N L}=$ $\operatorname{Var}\left[\underline{n}_{N L}\right]=a_{N L} P^{3}$ is the NLI power, obtained from a first-order regular perturbation [2,4]. The main goal of this paper is to provide a general analytical expression of the NLI coefficient $a_{N L}$, valid for dominant IFWM. Such an expression will be used to analytically cross-validate recent simulation results on nonlinear threshold (NLT) [5].

\section{Nonlinear Threshold}

We define the constrained NLT at reference $B E R_{0}$ (i.e., at its corresponding format-dependent SNR $S_{0}$ ) as the transmitted power $P_{N L T}$ yielding the maximum of the "bell-curve" $S$ versus $P$, where the maximum value is constrained to $S_{0}$. Maximization of (1) with ASE noise adjusted such that the top value is $S=S_{0}$ yields [2]

$$
P_{N L T}=\frac{1}{\left(3 S_{0} a_{N L}\right)^{1 / 2}}
$$

and depends only on $S_{0}$ and $a_{N L}$. It has been shown that the model (1), at the top $S$ value, yields an SNR penalty with respect to linear propagation of $1.76 \mathrm{~dB}[2,3]$. We can prove that the $1 \mathrm{~dB}$ NLT $P_{1}$, i.e., the transmitted power needed to achieve $S_{0}$ with $1 \mathrm{~dB}$ of SNR penalty, is $1.05 \mathrm{~dB}$ smaller than $P_{N L T}$. $P_{1}$ corresponds to the NLT simulated in [5] that we wish to double-check with our theory. 


\section{Nonlinear Interference coefficient}

We now describe a procedure to derive closed-form analytical expressions of the NLI coefficient $a_{N L}$. The NLI on each polarization tributary $(i=x$ or $y)$ can be obtained from a first-order regular perturbation as $[6,7]$ :

$$
n_{N L, i}=j \sqrt{P} \Phi_{N L} \iint_{-\infty}^{\infty} \eta\left(t_{1} t_{2}\right) U_{i}\left(t+t_{1}\right) U_{i}\left(t+t_{1}+t_{2}\right) U_{i}\left(t+t_{2}\right) d t_{1} d t_{2}
$$

where: the nonlinear phase is $\Phi_{N L} \triangleq P \int_{0}^{L} \gamma(s) G(s) \mathrm{d} s$, with $\gamma$ the fiber nonlinear coefficient and $G(s)$ the power gain at coordinate $s ; \eta\left(t_{1} t_{2}\right)$ is the time-domain kernel (time is normalized to the symbol time $1 / R$, where $R$ is the baud-rate), whose $2 \mathrm{D}$ Fourier transform is

$$
\tilde{\eta}(w) \triangleq \frac{\int_{0}^{L} \gamma(s) G(s) \mathrm{e}^{-j C(s) w} \mathrm{~d} s}{\int_{0}^{L} \gamma(s) G(s) \mathrm{d} s}
$$

where: $w=\omega_{1} \omega_{2} ; L$ is the total link length; and the normalized cumulated dispersion (NCD) is $C(s)=-R^{2} \int_{0}^{s} \beta_{2}(z) \mathrm{d} z$, where $\beta_{2}$ is the fiber chromatic dispersion, and zero dispersion slope is assumed. For a linear digital modulation we have $U_{i}(t)=\sum_{k=-\infty}^{\infty} s_{k} p(t-k)$ where $s_{k}$ is the complex information symbol (on polarization $i$ ) transmitted in the $k$-th symbol interval, and $p(t)$ is the supporting pulse. As done in [6,7], when the time-domain kernel is much broader than the symbol time and thus quasi-constant over squares of size 1 in the normalized time plane $\left(t_{1}, t_{2}\right)$, then the NLI term in (3), for a link with spans much longer than $1 / \alpha$ and lumped amplification, simplifies to $n_{N L, i}=c_{N L} P^{3 / 2}$, with

$$
c_{N L}=j \frac{\gamma}{\alpha} N \sum_{m, n, l} s_{m} s_{n} s_{l}^{*} \eta((m-l)(n-l))
$$

where the summation accounts for IFWM terms, i.e., is over all $m, n, l$ such that $m+n=l$, with $m \neq l, n \neq l$. The NLI power in (1) comes from both polarizations and is $N_{N L} \triangleq \eta_{p} E\left[\left|n_{N L}\right|^{2}\right]=\eta_{p} E\left[\left|c_{N L}\right|^{2}\right] P^{3}$, where $\eta_{p}=2$ for independent NLI from each polarization. Thus $a_{N L}=\eta_{p} E\left[\left|c_{N L}\right|^{2}\right]$, where the expectation is taken over the random symbols. For any modulation format with $E\left[s_{k}\right]=0$ and $E\left[\left|s_{k}\right|^{2}\right]=1$, we get

$$
a_{N L}=\eta_{p}\left(\frac{\gamma}{\alpha} N d_{f}\right)^{2} 2 \sum_{p=1}^{\infty} \sum_{q=1}^{\infty}|\eta(p q)|^{2}
$$

where $p \triangleq n-l, q \triangleq m-l$, and $d_{f}=2$ is the degeneracy factor. The time kernel magnitude decreases and eventually vanishes after an "effective" time duration $\tau_{M}$. Since each $|\eta(p q)|^{2}$ in the double summation in (5) is actually an approximation of the double integral of the kernel over a square of edge 1 centered at the point $(p, q)$, we can approximate the double summation as a double integral over the domain $\mathscr{D}$ of the $\left(t_{1}, t_{2}\right)$ plane delimited by the hyperbola $t_{1} t_{2}=\tau_{M}$, the vertical line passing through $t_{1}=1 / 2$, and the horizontal line passing through $t_{2}=1 / 2$. We can thus upper-bound the coefficient as

$$
a_{N L} \leq \eta_{p}\left(\frac{\gamma}{\alpha} N d_{f}\right)^{2} 2 \ln \left(4 \tau_{M}\right)\left[\int_{0}^{\infty}|\eta(\tau)|^{2} \mathrm{~d} \tau\right]
$$

and what we need is an expression of the kernel duration $\tau_{M}$, and of the above integral of the kernel magnitude. We may choose $\tau_{M} \triangleq \mu \tau_{r m s}$ for some positive multiplier $\mu$ of the r.m.s. width $\tau_{r m s}^{2}=\int_{-\infty}^{\infty} \tau^{2}|\eta(\tau)|^{2} \mathrm{~d} \tau / \int_{-\infty}^{\infty}|\eta(u)|^{2} \mathrm{~d} u$. We chose $\mu=1.5$ in all numerical results. Now, an analytical expression of the time kernel is not known even for the simplest links, except for lossless links [7]. However, there is a nice trick. For every optical link, both with and without dispersion management, a physically meaningful function is the power-weighted dispersion distribution (PWDD) $J(c)$, representing signal power versus $\mathrm{NCD} c$, which was shown to be the inverse 1D-Fourier transform: $J(c)=\mathscr{F}^{-1}[\tilde{\eta}(w)]$ [7]. One also has that: $\eta(\tau)=\mathscr{F}^{-1}\left[\frac{1}{|\omega|} J\left(\frac{1}{\omega}\right)\right]$, where $\tau=t_{1} t_{2}[6,7]$. Because of the Fourier relationship between $J(c)$ and $\eta(\tau)$, we can prove that $2 \int_{0}^{\infty}|\eta(\tau)|^{2} d \tau=\int_{-\infty}^{\infty} J^{2}(c) d c$, and that $\tau_{r m s}^{2}=\int_{-\infty}^{\infty}\left[J(c)+c J^{\prime}(c)\right]^{2} \mathrm{~d} c / \int_{-\infty}^{\infty} J^{2}(c) \mathrm{d} c$, where $J^{\prime}(c)=\frac{d}{d c} J(c)$. Hence, $a_{N L}$ in (6) can be expressed solely in terms of integrals of $J(c)$. Note also that it applies to any zero-mean modulation format. We managed to get closed-form expressions of the $a_{N L}$ upper-bound (6) for several links of interest. For instance, for NDM links we got for $N \gtrsim 5$ :

$$
a_{N L} \leq \eta_{P}\left(\frac{\gamma}{\alpha}\right)^{2} \frac{N}{\pi \mathscr{S}} \ln \left(\frac{4 \mu}{\sqrt{5}}(\alpha \ell N)^{2} \mathscr{S}\right)
$$

where $\ell$ is span length, and $\mathscr{S} \triangleq \frac{\left|\beta_{2}\right|}{\alpha} R^{2}$ is fiber strength. Note the similarity of this expression with that of a NyquistWDM NDM system derived in [3] using a frequency-domain approach. The major difference is the $N \log N$ scaling law in the IFWM-dominated regime, as opposed to the $\operatorname{simpler} N$ scaling when presumably cross-nonlinearities dominate. 

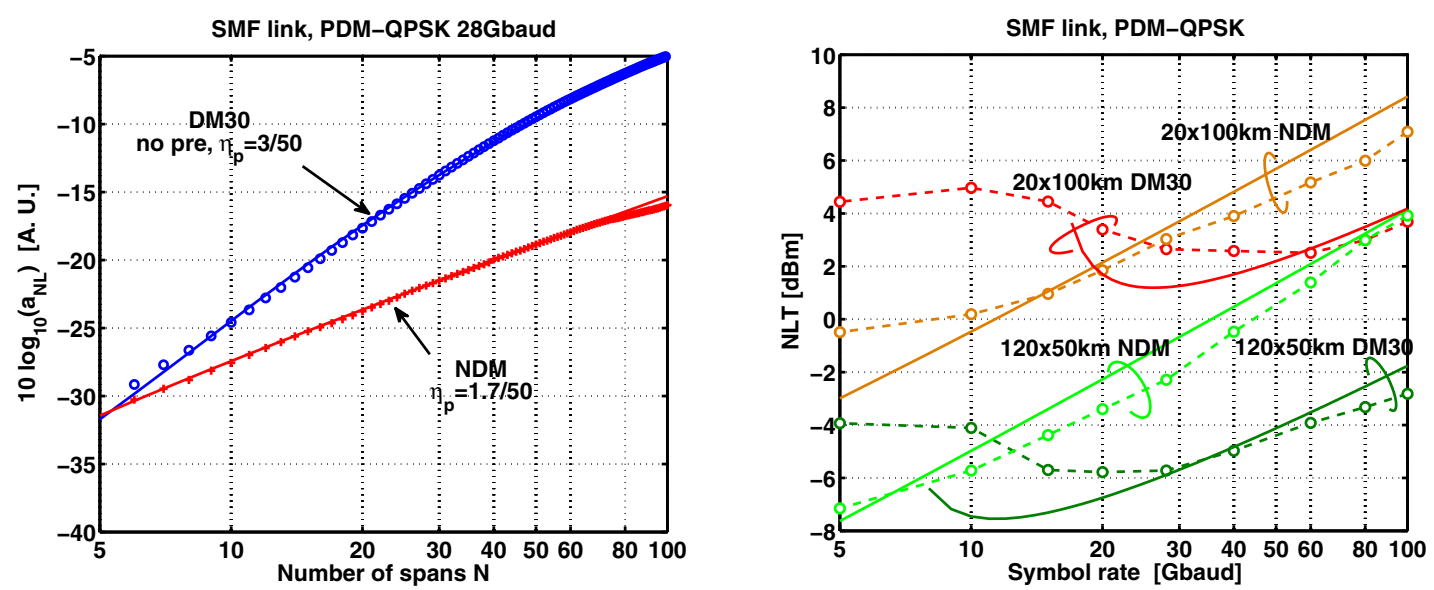

Figure 1. (Left) $a_{N L}[\mathrm{~dB}]$ versus spans $N$ from eq. (6) (solid) and simulations (symbols). PDMQPSK on $N \times 100 \mathrm{~km}$ SMF links, R=28 Gbaud. (Right) 1dB NLT vs. symbol rate $R$ for: i) theory $P_{1}=P_{N L T}-1.05 \mathrm{dBm}$ (solid, eq. (2)); ii) simulations from [5]. DM30 = DM with $30 \mathrm{ps} / \mathrm{nm}$ RDPS.

\section{Results}

Fig. 1(left) shows a plot of the $a_{N L}$ formula (6) versus number of spans $N$ (solid), and numerically simulated values (symbols), for a 28 Gbaud polarization-division multiplexed quadrature phase shift keying (PDM-QPSK) coherent format over single mode fiber (SMF, $\beta_{2}=-21 \mathrm{ps}^{2} / \mathrm{nm}$ ) for an $N \times 100 \mathrm{~km}$ link, both NDM and DM with $30 \mathrm{ps} / \mathrm{nm} / \mathrm{km}$ (DM30) of residual dispersion per span (RDPS) and no pre-compensation. A fitting factor $\eta_{p}=3 / 50$ was used for $\mathrm{DM}$, and $\eta_{p}=1.7 / 50$ for NDM. We appreciate the match of theory and simulation, as well as the announced $N \log N$ scaling law in the NDM case. The perceived NDM slope over a 50 span range is $\sim 1.25 \mathrm{~dB} / \mathrm{dB}$ as in [1], although restricting the range to the first 15 spans gives $\sim 1.35 \mathrm{~dB} / \mathrm{dB}$, as we experimentally verified in a companion study. NLI grows faster in the DM case: $a_{N L}$ has an initial slope of $\sim 2 \mathrm{~dB} / \mathrm{dB}$ and then bends at larger $N$.

Fig. 1(right) shows the $1 \mathrm{~dB}$ NLT at $B E R_{0}=10^{-3}$ versus baud-rate for a PDM-QPSK format for both NDM, and a DM30 link with optimized pre-compensation, both at $20 \times 100 \mathrm{~km}$ and at $120 \times 50 \mathrm{~km}$ distance. Symbols refer to singlechannel simulation results taken from [5], solid lines to the formula $P_{1}=P_{N L T}-1.05 \mathrm{dBm}$ using (2) and the same $\eta_{p}$ fitting factors as in Fig. 1(left). While for DM links theory only captures the general trend versus $R$ with major discrepancies at lower $R$ where IFWM is not dominant, the match in NDM links (optimized at 28 Gbaud through the fitting factors $\eta_{p}$ ) is more reasonable and improves as the number of spans $N$ increases.

\section{Conclusions}

We have provided a new model of NLI in IFWM dominated links, which reasonably models NDM links, as well as high baud-rate DM links. Such a model provides a quick qualitative tool to compare transmission link parameters in terms of their impact on received SNR.

\section{References}

1. A. Carena, G. Bosco, V. Curri, P. Poggiolini, M. Tapia Taiba, F. Forghieri, "Statistical Characterization of PM-QPSK Signals after Propagation in Uncompensated Fiber Links, “ECOC'10, P4.07.

2. G. Bosco et al., "Performance Prediction for WDM PM-QPSK Transmission over Uncompensated Links", OFC'11, OThO7.

3. P. Poggiolini et al., "Analytical Modeling of Non-Linear Propagation in Uncompensated Optical Transmission Links", Photon. Technol. Lett., to appear, 2011.

4. E. Grellier et al., "Quality Parameter for Coherent Transmissions with Gaussian-distributed Nonlinear Noise", Optics Express, submitted, 2011.

5. A. Bononi, P. Serena, N. Rossi, "Transmission Limitations due to Fiber Nonlinearity," OFC'11, OWO7.

6. A. Bononi, P. Serena and M. Bertolini, "Unified Analysis of Weakly-Nonlinear Dispersion-Managed Optical Transmission Systems from Perturbative Approach," Comptes Rendus - Physique, vol. 9, 2008, pp. 947-962.

7. X. Wei, "Power-weighted dispersion distribution function for characterizing nonlinear properties of long-haul optical transmission links," Opt. Lett., vol. 31, no. 17, pp. 2544-2546, Sept. 2006. 